\title{
Structuring and Fabrication of Sensors Based on LTCC (Low Temperature Co-fired Ceramic) Technology
}

Hansu Birol, Thomas Maeder, Caroline Jacq, Giancarlo Corradini, Marc Boers, Ingo Nadzeyka, Sigfrid Straessler \& Peter Ryser

Swiss Federal Institute of Technology, Lausanne - EPFL

Laboratory for Production of Microtechnologies - LPM

Thick-film Group

$$
\text { lpmwww.epfl.ch }
$$




\section{PURPOSE OF THE}

\section{Introduction to LTCC Technology:}

General aspects

Sensor applications

Fabrication techniques for sensors: Major problems encountered

Methods for structuring

Methods for reducing deformation

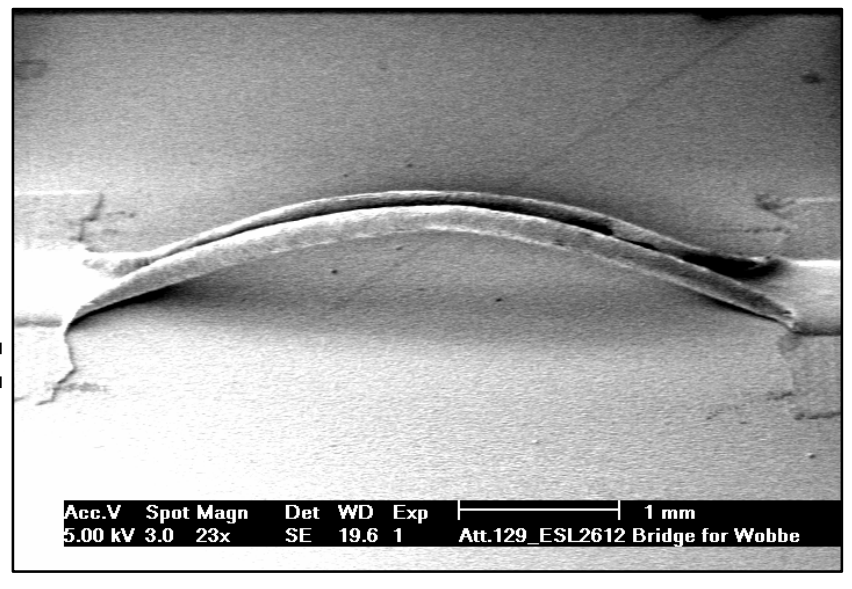

Fabricated sensors: Completed devices

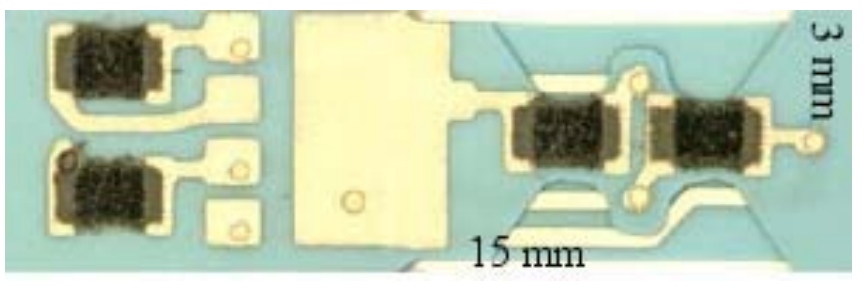




\section{AN OVERVI EW}

LTCC for Wireless Applications

Base Station Amplifier Modules

Transmitters and Receivers

Handset Power Amplifiers

Low Noise Amplifiers

Voltage Control Oscillators

Mixers

Filters

Power Splitters and Combiners

Matching Networks

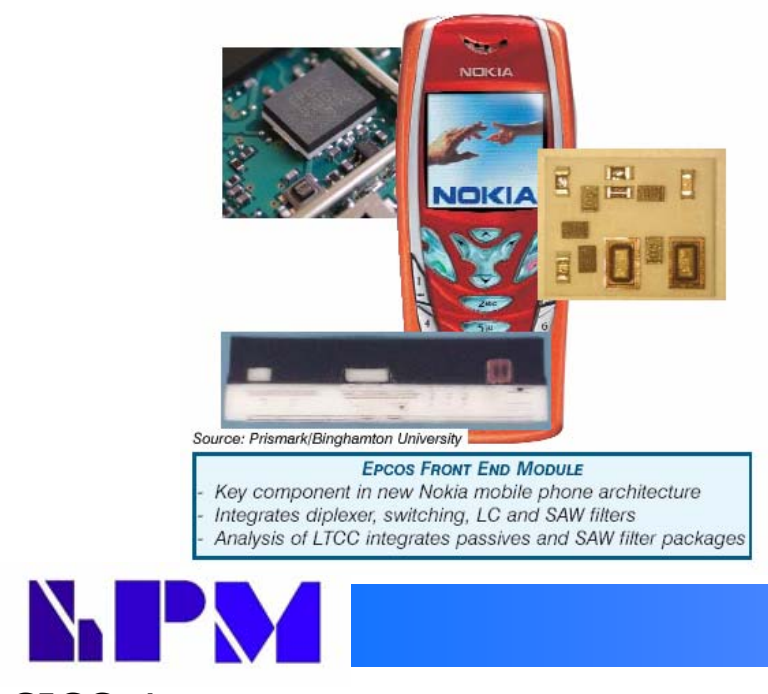

CICC-4
LTCC in the Automotive Industry

Engine Management Systems

Gearbox Management Systems

Anti-Lock Braking Systems

Global Positioning Systems

Gas Discharge Lamp Controllers

Ignition Modules

Sensor Modules

Bluetooth-Module @ 2,45 GHz

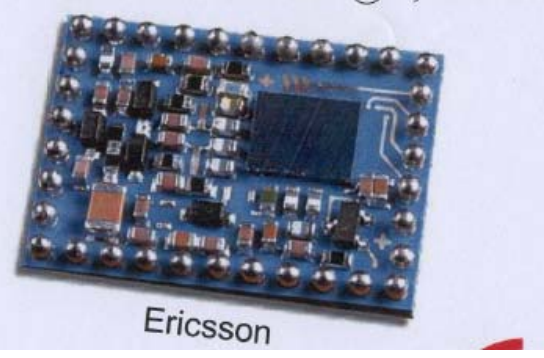

Hansu BIROL
LTCC in Military \& Space

Environments

Transmitters/Receivers

Phased Array Radar

Amplifiers

Filters

Converters

Power Drivers

Sensors

Source: C-MAC Micro-technology

And recently for ...
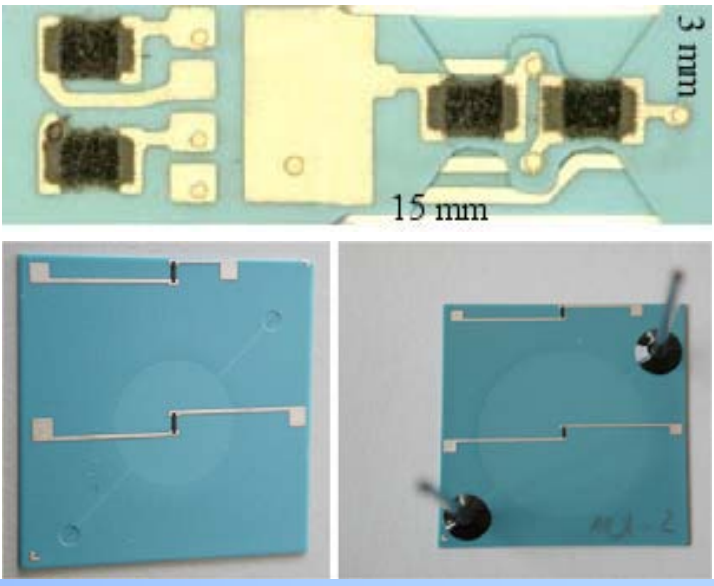

October 23-26, 2005 -CHINA 


\section{NTRODUCTI ON: LTCC MATERI ALS SYSTEM}

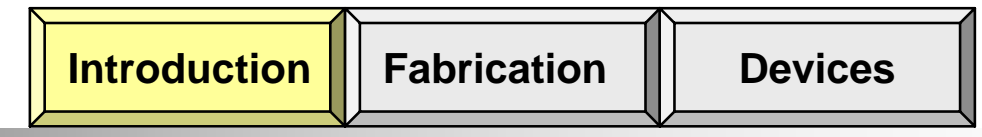

(4)

ÉCOLE POLYTECHNIOUE FÉDÉRALE DE LAUSANNE

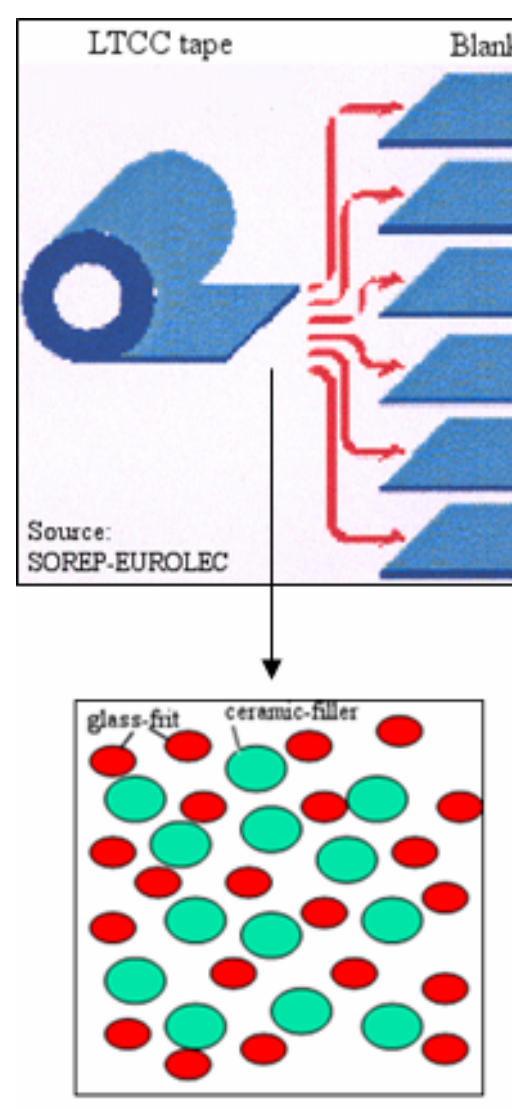

Glass-frit: low firing $\mathrm{T}$

Filler: dimensional stability

.. blended in organic-vehicle \& cast on mylar sheets
Blanking Via punching Via filling Conductor printing

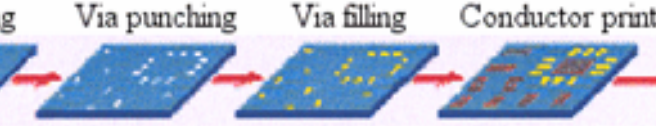

\section{.}




\section{ADVANTAGES OF LTCC FOR SENSOR APPLI CATI ONS}

\begin{tabular}{|l|l|l|}
\hline Introduction & Fabrication & Devices \\
\hline
\end{tabular}

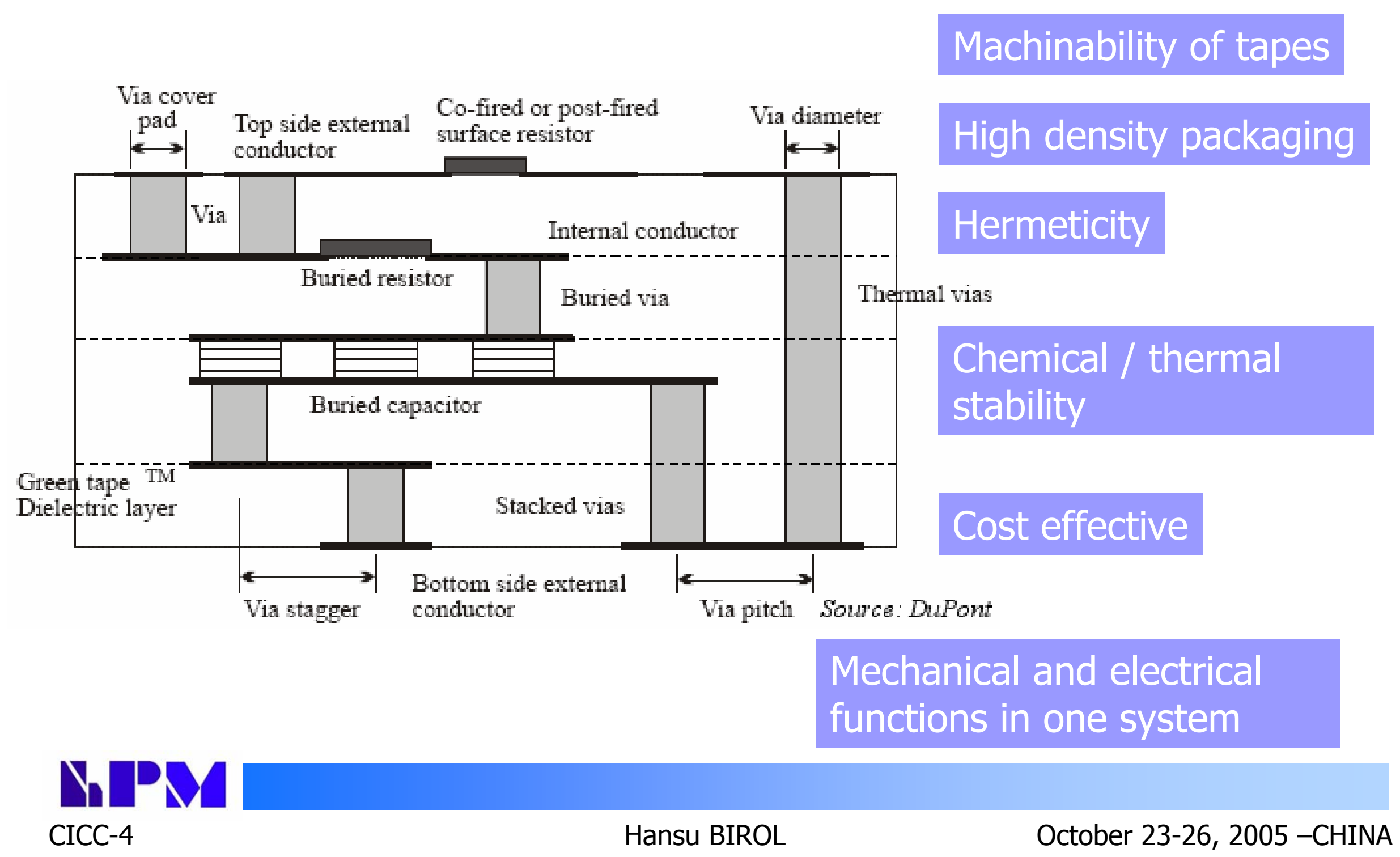




\section{FABRI CATI ON: DEFI NITION OF PROBLEMS I}

Sagging in cavities

Unsupported cavity is deformed during:

$\rightarrow$ Lamination (lamination stress)

$\rightarrow$ Sintering (over Tg)

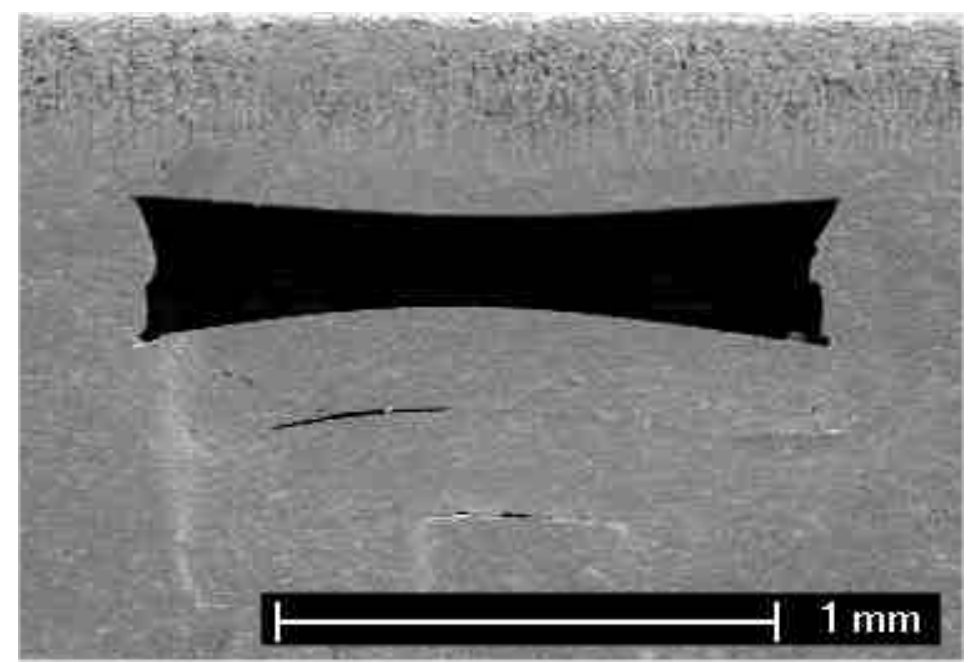

Delamination / Disintegration

Occurs due to:

$\rightarrow$ Poor lamination

$\rightarrow$ Geometrical constraints

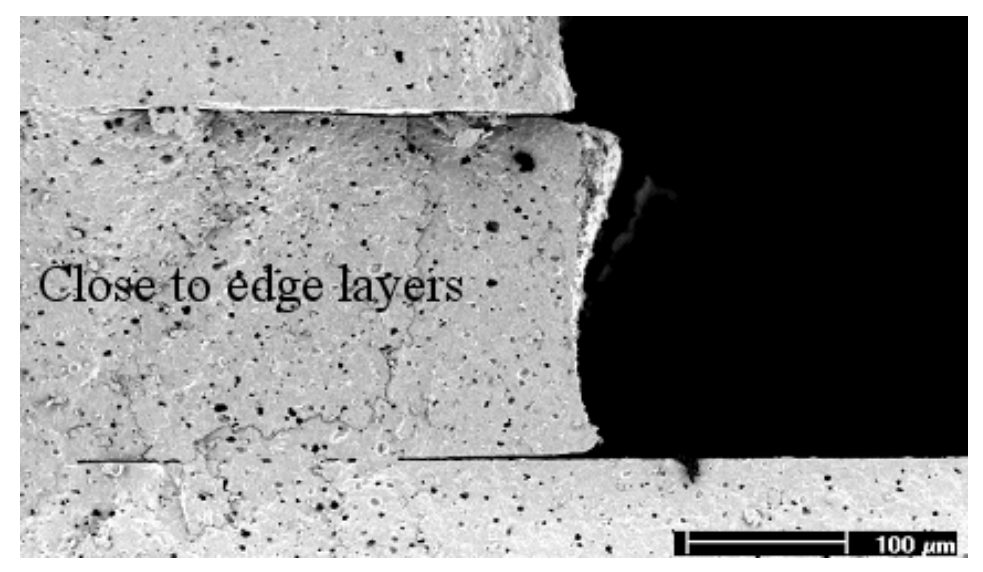




\section{FABRI CATI ON: DEFI NI TI ON OF PROBLEMS II}

\begin{tabular}{|l||l|l||}
\hline Introduction & Fabrication & Devices \\
\hline
\end{tabular}

Deformation of co-fired bodies (warpage, etc...) due to differential shrinkage

As a result of:

1. rapid sintering

2. higher extent of shrinkage

of thick-film components com-

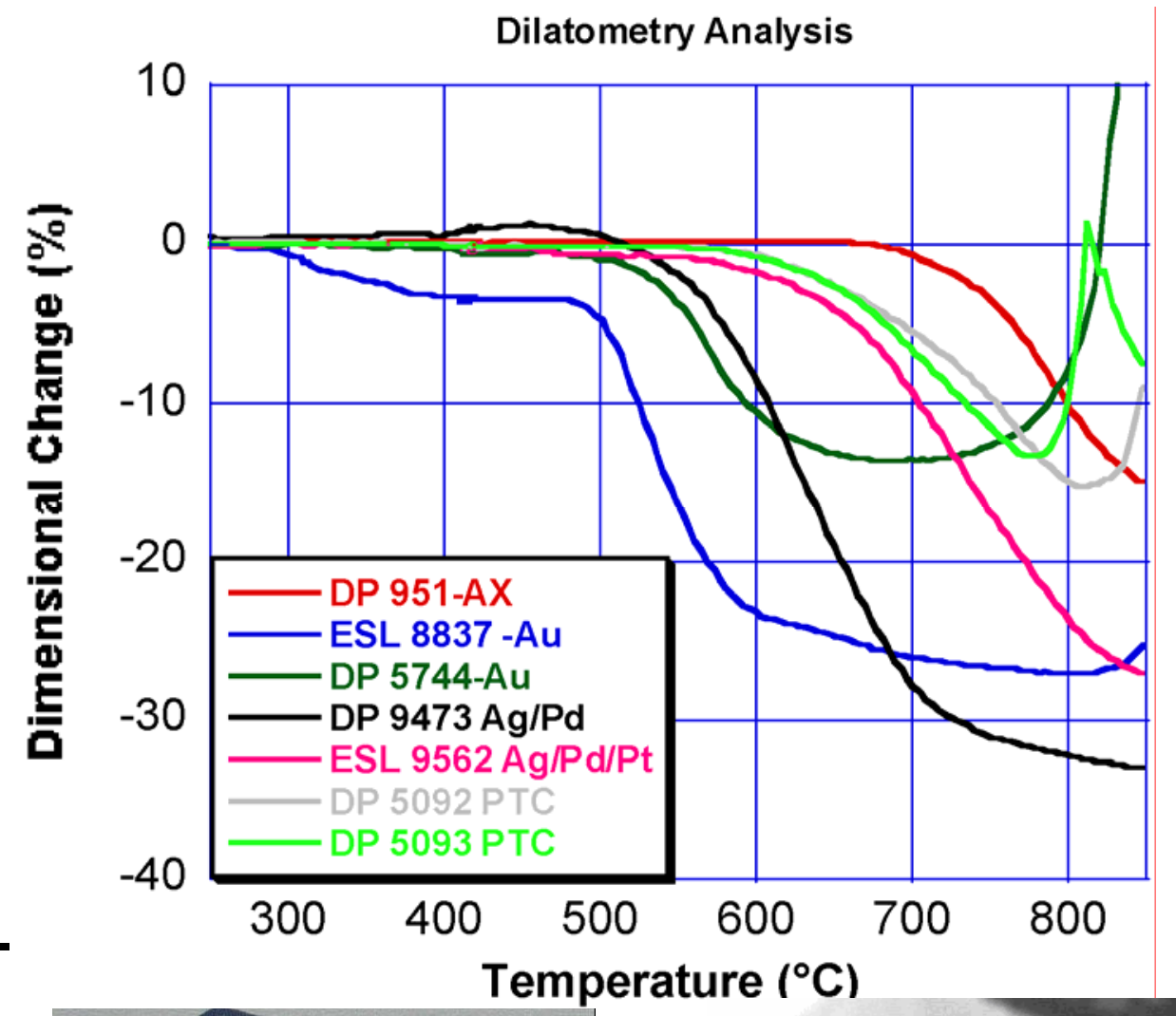
pared to LTCC
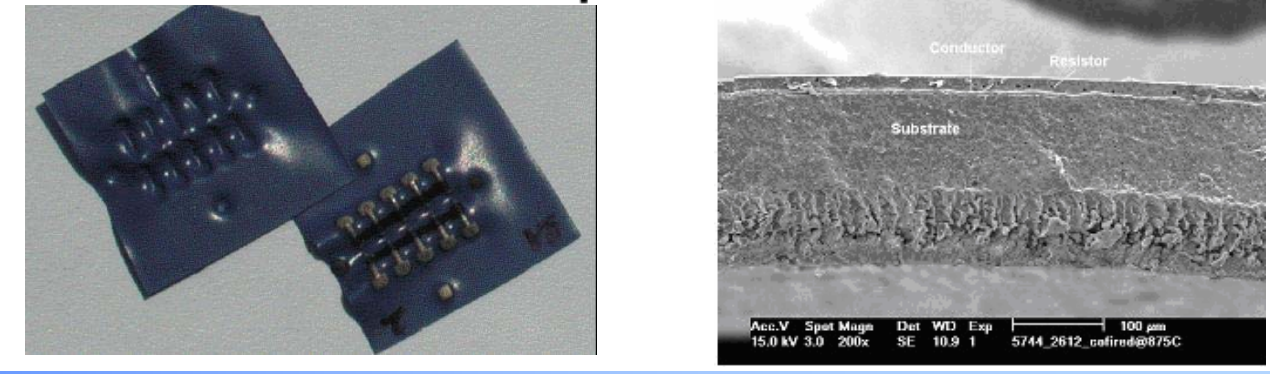


\section{SOLUTI ONS \\ DEVELOPED I \\ (for 3-D)}

\section{METHODS FOR STRUCTURING}

Utilization of temporary sacrificial layers, which are screenprinted \& laminated with LTCC to fabricate «sag-free \& wellintegrated 》

- micro-fluidic channels,

- cavities such as membranes,

\begin{tabular}{|l|l|l|l|}
\hline \multicolumn{1}{|c|}{ Product } & Function & \multicolumn{1}{c|}{ Specification } & \multicolumn{1}{c|}{ Supplier } \\
\hline \multirow{2}{*}{ Graphite } & \multirow{2}{*}{ Sacrificial } & $\mathrm{d}_{50}: 1-2 \mu$ (used lot) & Aldrich, 28,286-3 \\
\cline { 3 - 4 } & & $\mathrm{d}_{50}: 11 \mu$ & $\mathrm{KS} 25$ \\
\cline { 3 - 4 } & & $\mathrm{d}_{50}: 15.3 \mu$ & KS5-25 \\
\hline $\begin{array}{l}\text { Ethyl } \\
\text { cellulose }\end{array}$ & Binder & control of rheology & Aldrich, 43,383-7 \\
\hline Terpineol & Solvent & slurry viscosity & Fluka, 86480 \\
\hline $\begin{array}{l}\text { Acetyl } \\
\text { acetone }\end{array}$ & Dispersant & dispersing additive & Sigma-Aldrich, P775-4 \\
\hline
\end{tabular}
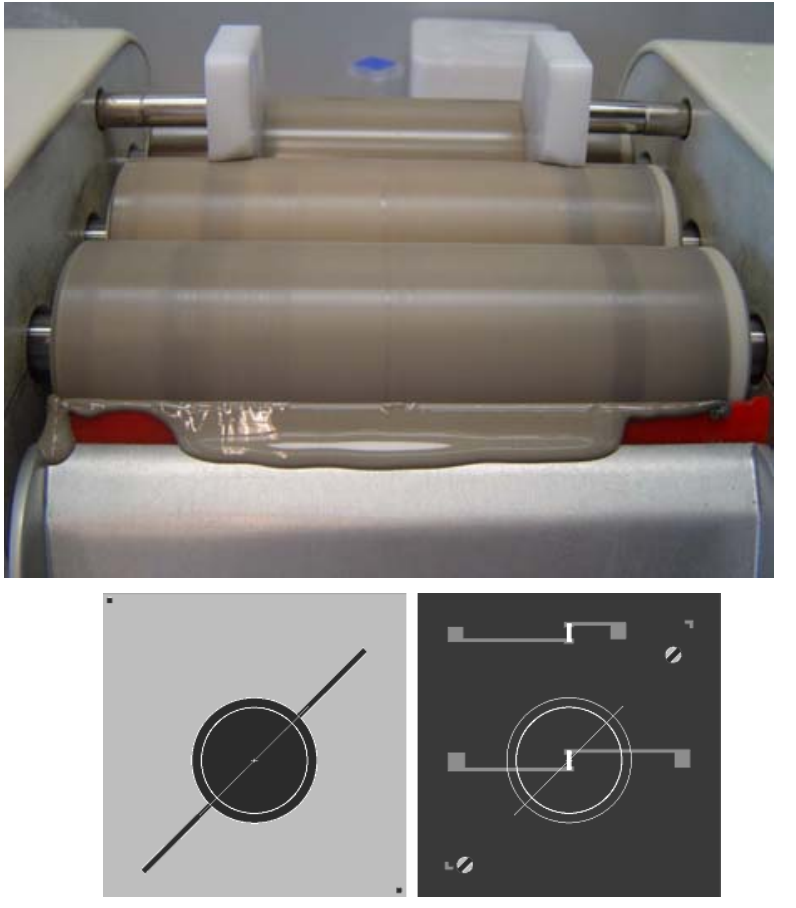


\section{SOLUTI ONS DEVELOPED I I (for flatness)}

\section{Introduction | Fabrication}

METHODS FOR REDUCING « DIFFERENTIAL SHRINKAGERELATED » DEFORMATION

Modifying commercial thickfilm components by selected additives to

- shrinkage match paste with

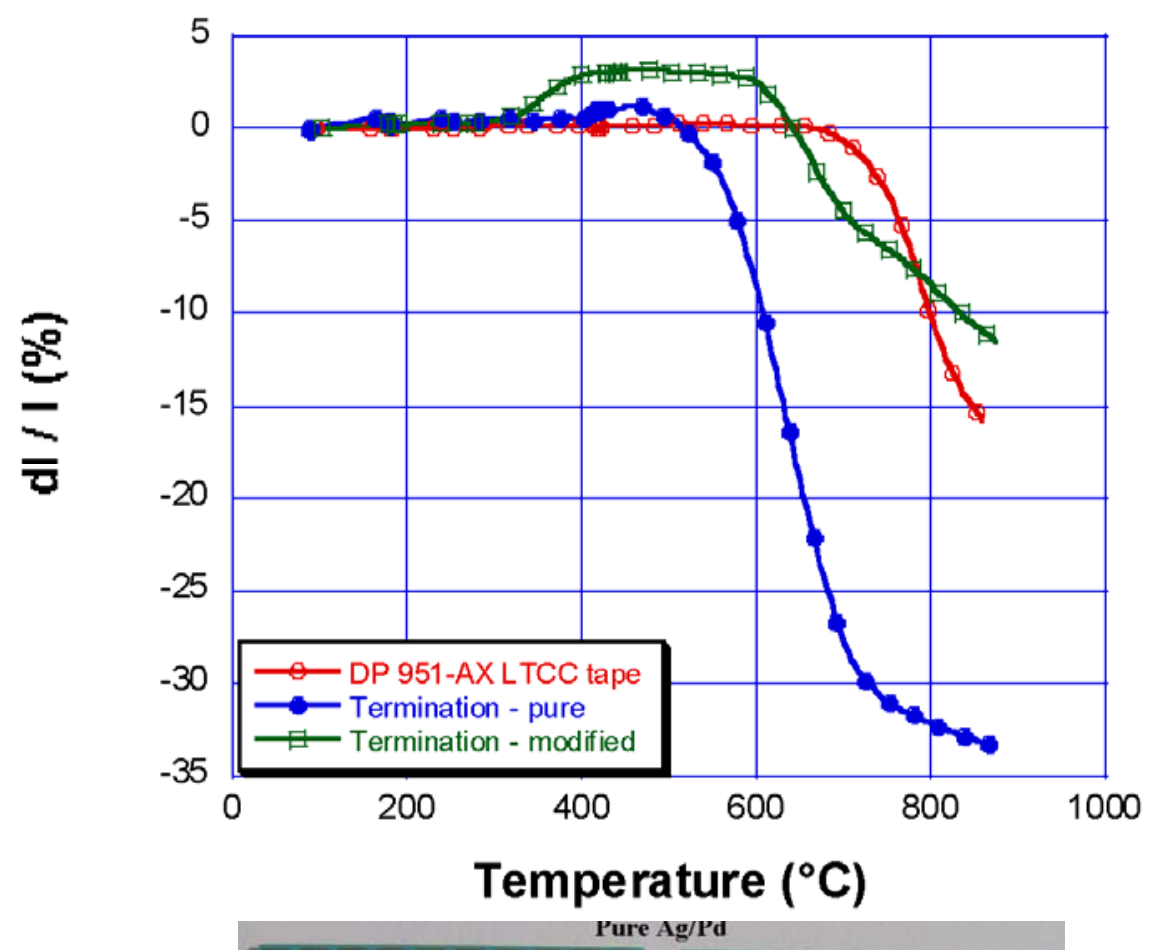

LTCC,

- reduce the overall shrinkage of the paste,

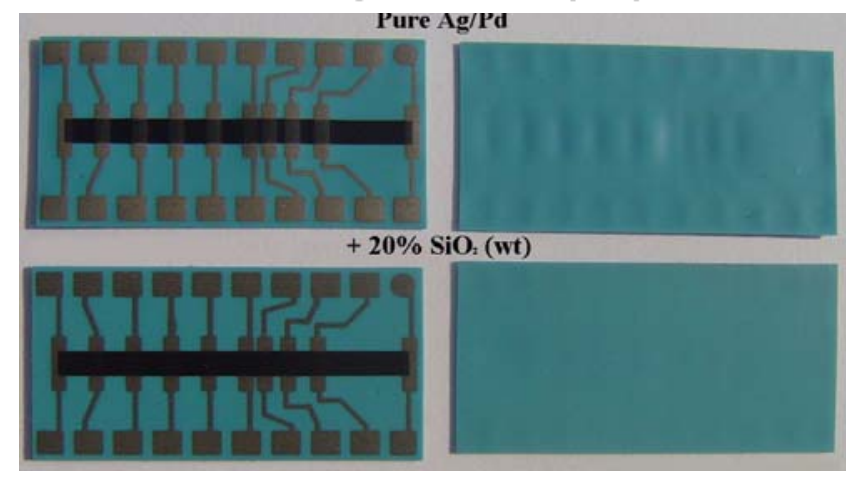




\section{I NCLI NATI ON SENSOR}

\section{LTCC: an excellent substrate for hot-spot application $(\lambda \sim 2-3 \mathrm{~W} / \mathrm{m} . \mathrm{K})$}
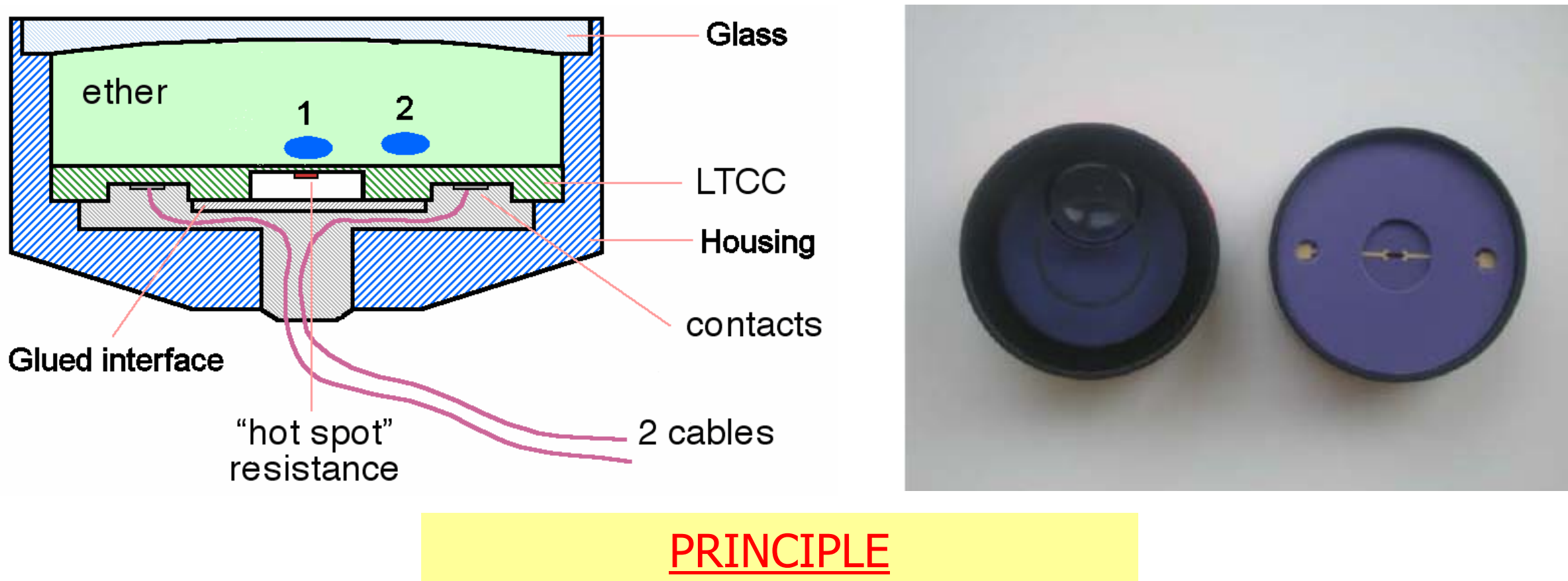

\section{PRINCIPLE}

Detection of heat loss from the PTC resistor to the surroundings (bubble surface / liquid) 


\section{NCLI NATI ON SENSOR: RESULTS}

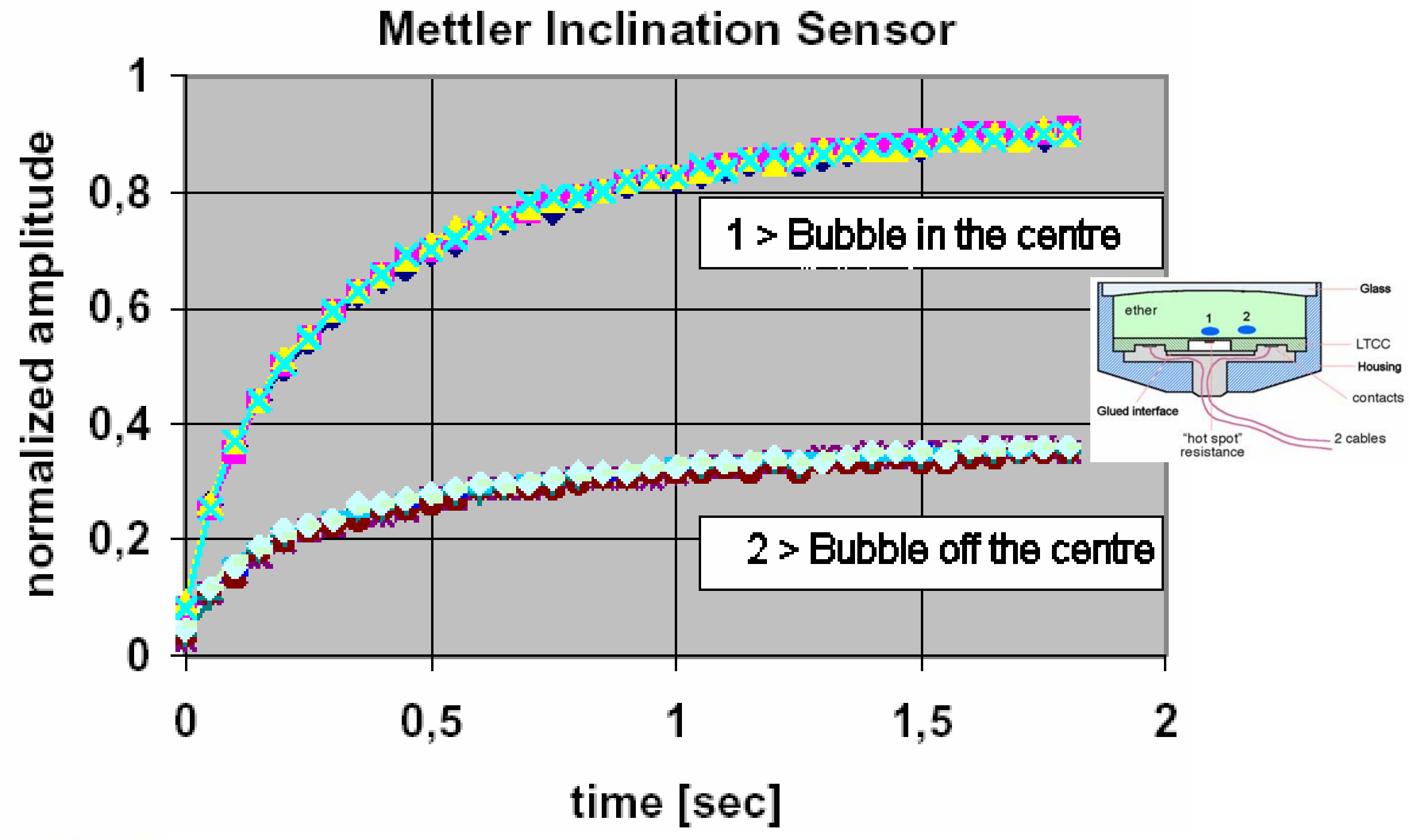




\section{MI LLI NEWTON FORCE SENSOR: MOTI VATI ON}

LTCC: an excellent substrate for force sensing

Maximum signal, $(\Delta R / R)$

Signal $=\varepsilon_{\max } \mathrm{G}_{\mathrm{f}}$

Maximum strain, $\varepsilon(\Delta l / l) \Longleftrightarrow \mathrm{E}=\sigma / \varepsilon$

Maximum stress, $\sigma_{\max }$

$$
\left.\begin{array}{l}
\mathrm{E}=\sigma / \varepsilon \\
\sigma_{\max }=(6 \mathrm{FL}) /\left(\mathrm{bh}^{2}\right)
\end{array}\right\} \varepsilon=(6 \mathrm{FL}) /\left(\mathrm{bh}_{\uparrow \uparrow}^{2}\right) \underset{\uparrow}{\mathrm{E}}
$$

\begin{tabular}{|c|c|c|}
\hline Properties & $\begin{array}{c}\text { Kyocera A-476 } \\
\mathbf{A l}_{\mathbf{2}} \mathbf{O}_{\mathbf{3}} \mathbf{( 9 6 \% )}\end{array}$ & $\begin{array}{c}\text { DuPont LTCC } \\
\mathbf{9 5 1} \text { (fired) }\end{array}$ \\
\hline Elastic modulus (GPa) & 330 & 152 \\
\hline Flexural strength (MPa) & 310 & 320 \\
\hline Available thickness (mm) & $0.25-1.00$ & $0.04-0.21$ \\
\hline
\end{tabular}

$$
\begin{gathered}
\varepsilon_{\mathrm{LTCC}} / \varepsilon_{\mathrm{Al} 2 \mathrm{O} 3}=\left(\mathrm{h}^{2}{ }_{\mathrm{Al} 2 \mathrm{O} 3} \mathrm{E}_{\mathrm{Al} 2 \mathrm{O} 3}\right) /\left(\mathrm{h}^{2}{ }_{\mathrm{LTCC}} \mathrm{E}_{\mathrm{LTCC}}\right) \\
\varepsilon_{\mathrm{LTCC}} / \varepsilon_{\mathrm{A} 1203} \rightarrow \text { up to } \sim 70 \text { times theoretically }
\end{gathered}
$$




\section{LLI NEWTON FORCE SENSOR: FABRI CATI ON}

Introduction Fabrication

\section{CUTTING \& LAYOUT}
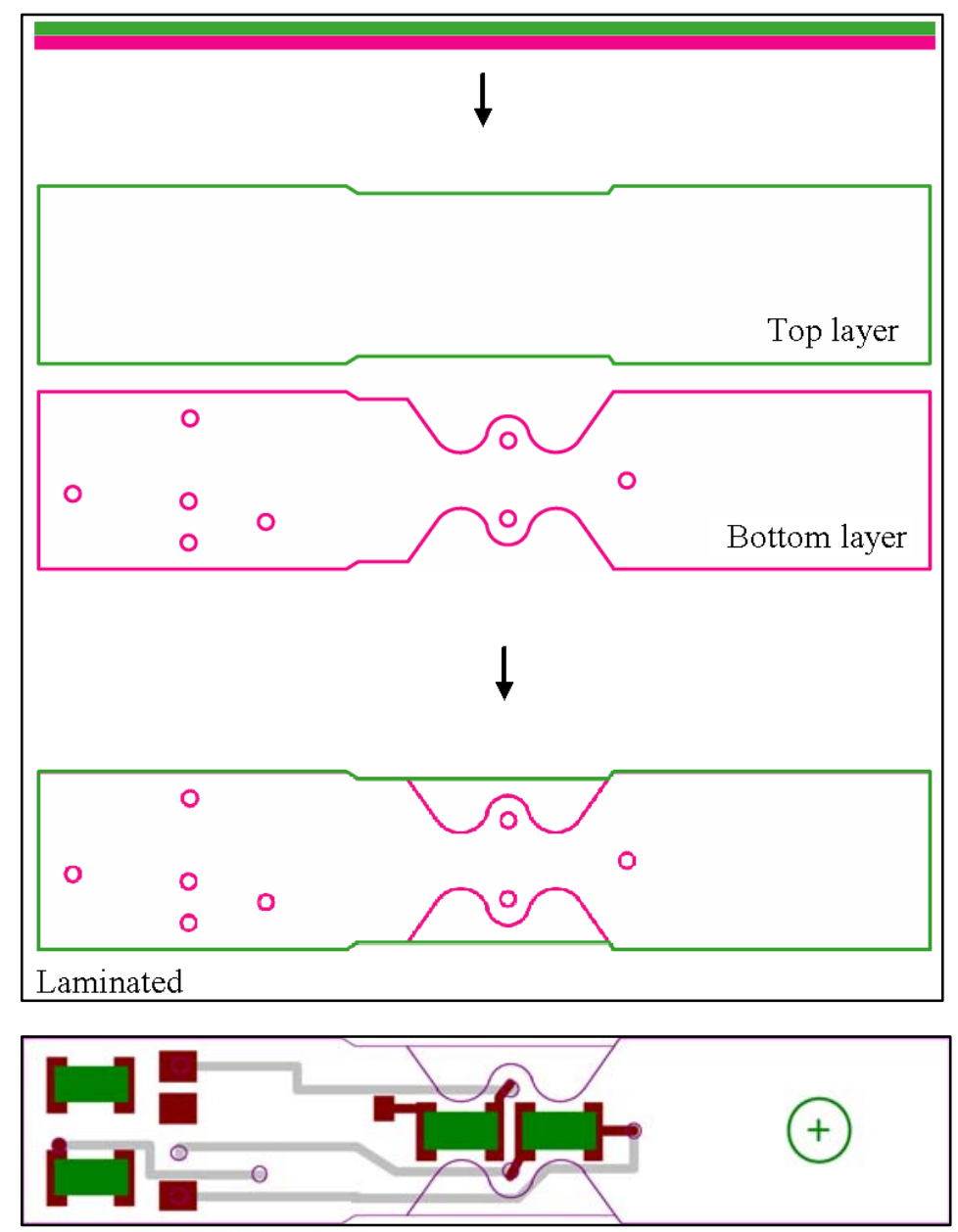

MPM

CICC-4
CO-FIRING
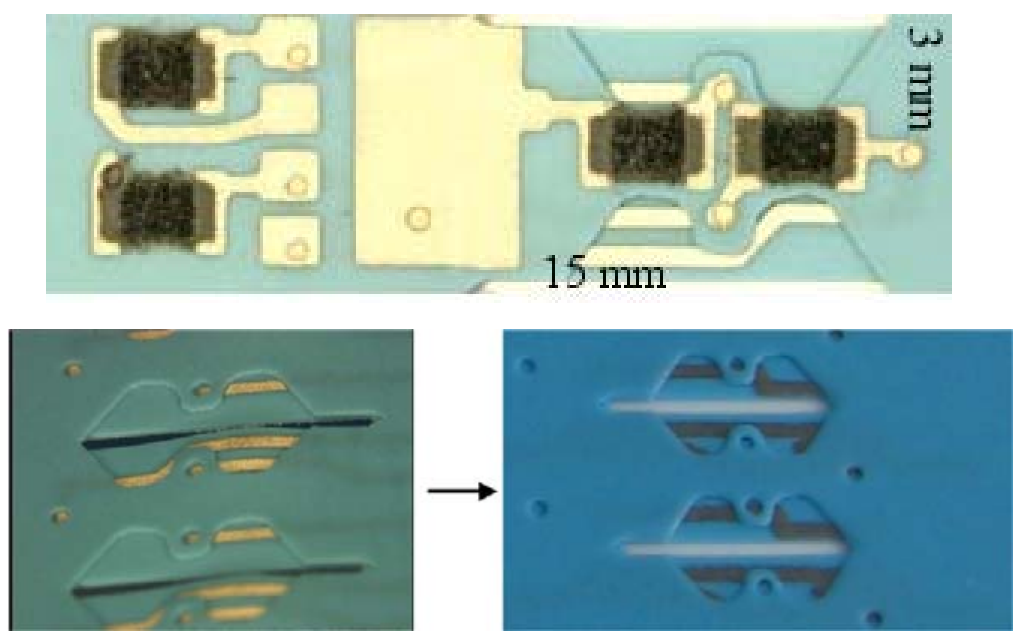

MEASUREMENT SET-UP

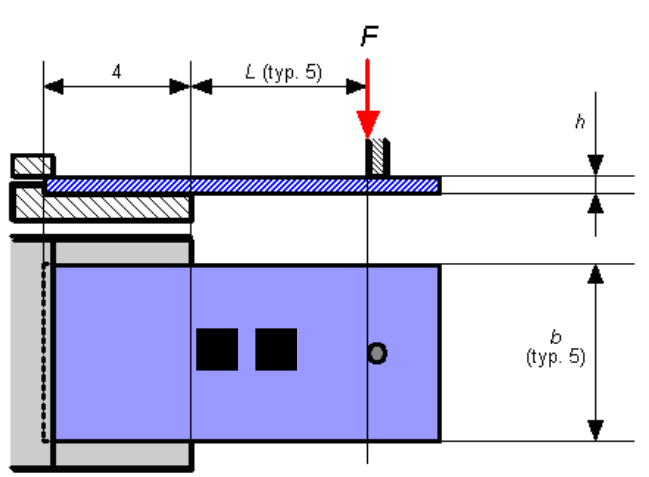

October 23-26, 2005 -CHINA 


\section{LLI NEWTON FORCE SENSOR: RESULTS}

\section{Performance of LTCC-based MFS}
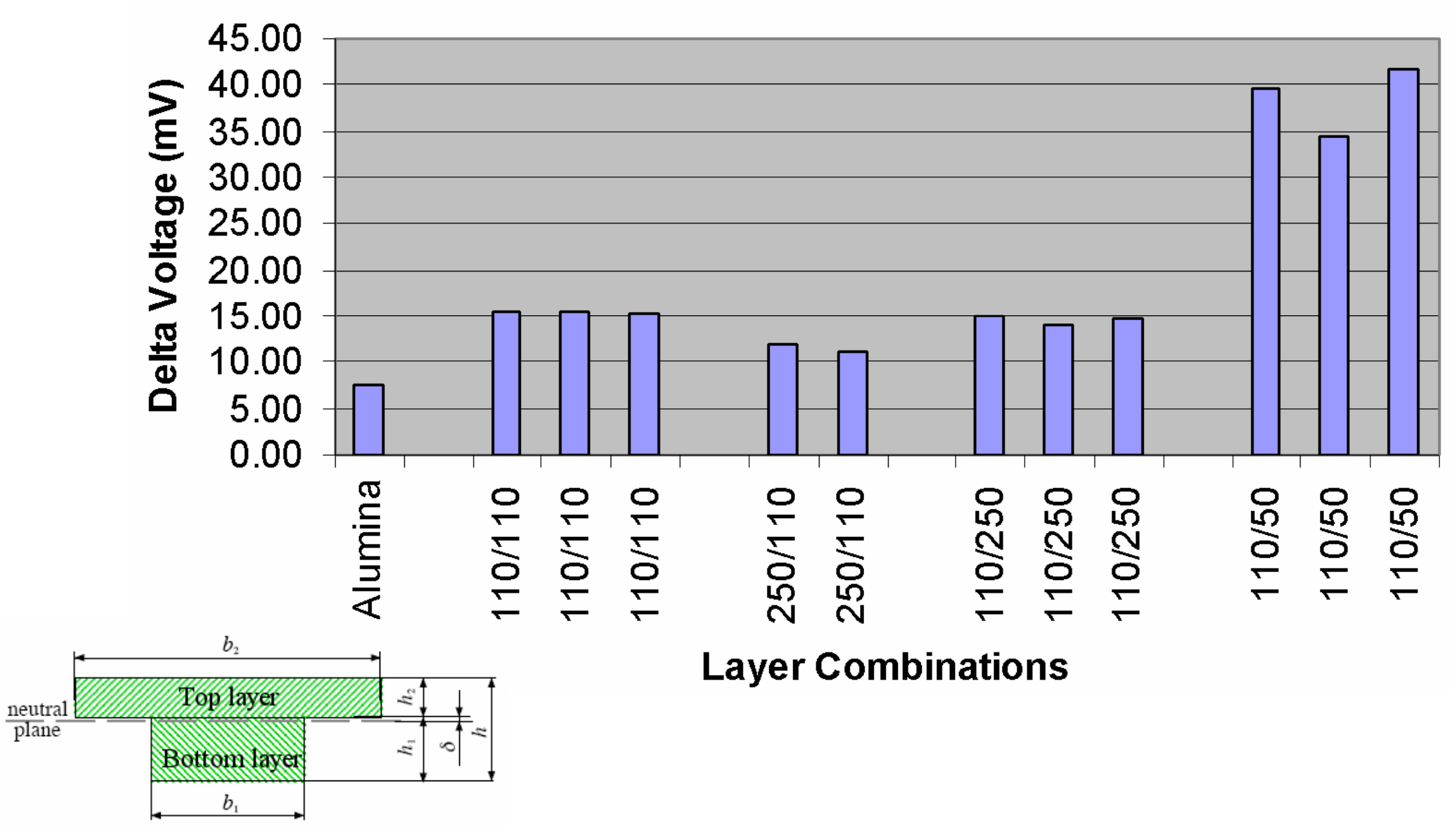


\section{MI CRO-FLUI DI C SENSOR (Pressure or heat conductivity)}

LTCC + sacrificial layers: an effective \& smart packaging approach
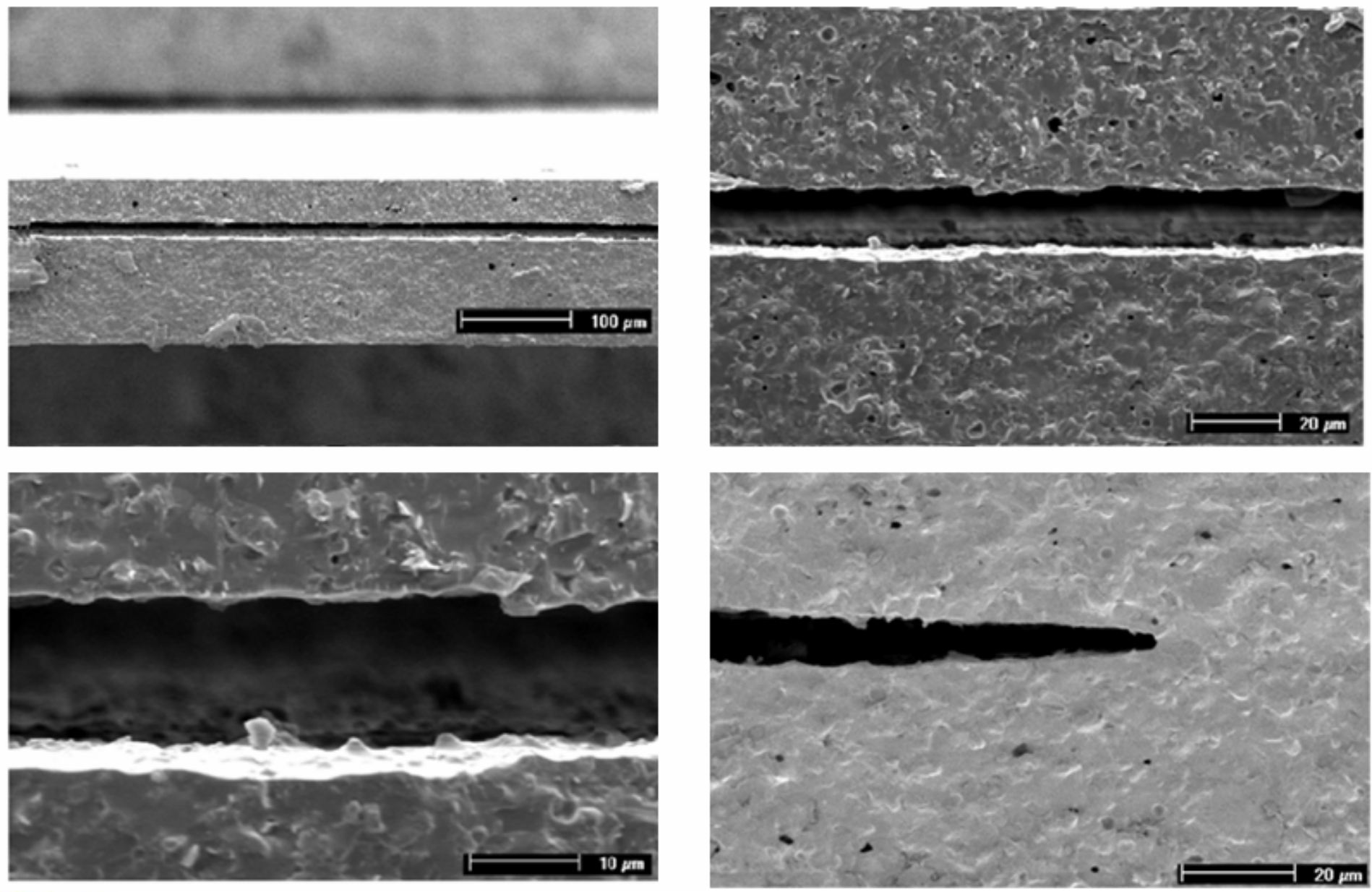


\section{CRO-FLUI DI C SENSOR: STRUCTURES}

Using the graphite-based sacrificial paste, membranes produced with

$\rightarrow$ thickness: of $40 \mu \mathrm{m}$

$\rightarrow$ diameter: $7,10,15, \underline{18 \mathrm{~mm}}$

$\rightarrow$ spacing: $(\underline{10-100 \mu m})$

Final membrane features dependent on graphite powder and LTCC properties

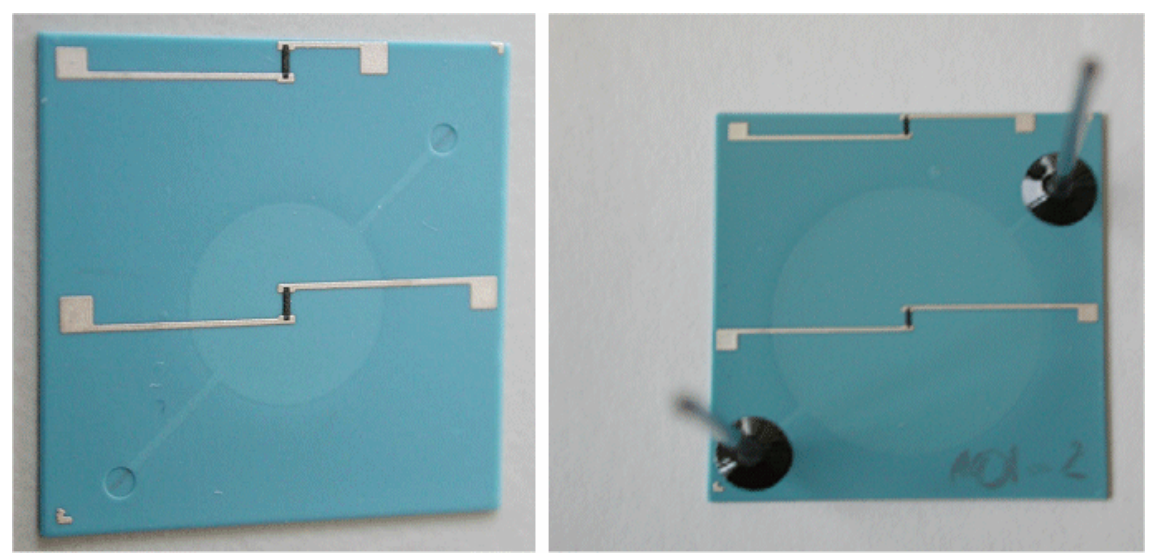

14mm-diameter-membrane with PTC resistors

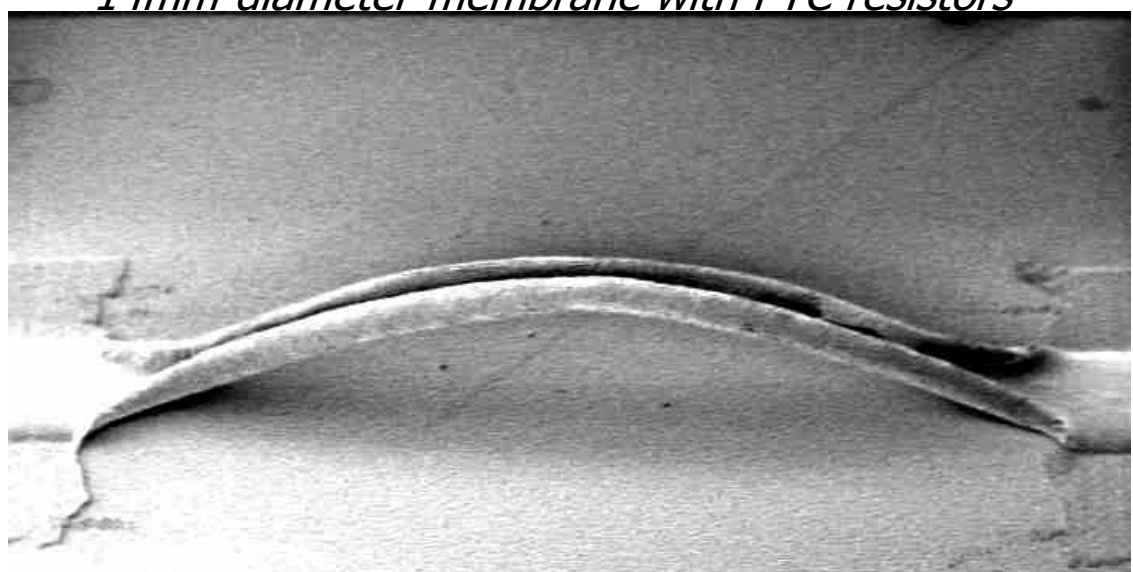

\begin{tabular}{lllll}
\hline Ace.V Spot Magn & Det & WD Exp \\
$5.00 \mathrm{kV}$ & $3.023 \mathrm{x}$ & SE & 19.61
\end{tabular}

$1 \mathrm{~mm}$
Att.129 ESL2612 Bridge for Wobhe

PTC free-hanging bridge on LTCC 
$\rightarrow$ LTCC Technology has been efficiently used for fabrication of sensor \& micro-fluidic devices such as:

$\rightarrow$ Structuration \& fabrication is basically limited by:

$\rightarrow$ Low sintering temperature, High packaging density, Thermal \& chemical stability Integrability with thick-films inclination, millinewton force, pressure sensors \& microfluidics the methods used for structuring and the materials compatibility issues

favors the application of the technology in multi-disciplinary areas very soon 